\title{
Critical functions of the polyamine putrescine for proliferation and viability of Leishmania donovani parasites
}

\author{
Jasmine Perdeh ${ }^{1} \cdot$ Brandon Berioso $^{1} \cdot$ Quintin Love $^{1} \cdot$ Nicole LoGiudice $^{1,2} \cdot$ Thao Linh Le $^{1,3} \cdot$ John P. Harrelson ${ }^{1}$. \\ Sigrid C. Roberts ${ }^{1}$ (D)
}

Received: 17 January 2019 / Accepted: 10 April 2019 / Published online: 16 April 2019

(c) The Author(s) 2019

\begin{abstract}
Polyamines are metabolites that play important roles in rapidly proliferating cells, and recent studies have highlighted their critical nature in Leishmania parasites. However, little is known about the function of polyamines in parasites. To address this question, we assessed the effect of polyamine depletion in Leishmania donovani mutants lacking ornithine decarboxylase $(\Delta o d c)$ or spermidine synthase ( $\Delta s p d s y n)$. Intracellular putrescine levels depleted rapidly in $\Delta o d c$ mutants and accumulated in $\Delta$ spdsyn mutants, while spermidine levels were maintained at low but stable levels in both cell lines. Putrescine depletion in the $\Delta o d c$ mutants led to cell rounding, immediate cessation of proliferation, and loss of viability, while putrescine-rich $\Delta$ spdsyn mutants displayed an intermediate proliferation phenotype and were able to arrest in a quiescent-like state for 6 weeks. Supplementation of $\Delta o d c$ mutants with spermidine had little effect on cell proliferation and morphology but enabled parasites to persist for 14 weeks. Thus, putrescine is not only essential as precursor for spermidine formation but also critical for parasite proliferation, morphology, and viability.
\end{abstract}

Keywords Leishmania $\cdot$ Polyamines $\cdot$ Putrescine $\cdot$ Gene deletion mutants $\cdot$ Proliferation $\cdot$ Viability

$\begin{array}{ll}\text { Abbreviations } & \\ \text { ADOMETDC } & \text { Adenosylmethionine decarboxylase } \\ \text { ARG } & \text { Arginase } \\ \text { DFMO } & \text { Difluoromethylornithine } \\ \text { DHS } & \text { Deoxyhypusine synthase } \\ \text { DOOH } & \text { Deoxyhypusine hydroxylase } \\ \text { ODC } & \text { Ornithine decarboxylase } \\ \text { PBS } & \text { Phosphate buffered saline } \\ \text { SPDSYN } & \text { Spermidine synthase } \\ \text { SPMSYN } & \text { Spermine synthase }\end{array}$

Handling Editor: E. Agostinelli.

Electronic supplementary material The online version of this article (https://doi.org/10.1007/s00726-019-02736-z) contains supplementary material, which is available to authorized users.

Sigrid C. Roberts

sroberts@pacificu.edu

1 Pacific University School of Pharmacy, Hillsboro, OR 97123, USA

2 Present Address: McKenzie Willamette Medical Center, Springfield, OR 97477, USA

3 Present Address: Washington State University College of Pharmacy, Spokane, WA 99202, USA
TCA Trichloroacetic acid

TRYS Trypanothione synthetase-amidase

\section{Introduction}

Parasites of the genus Leishmania cause a variety of devastating diseases in humans and domestic animals worldwide. The spectrum of leishmaniasis ranges from cutaneous ulcerative lesions to fatal visceralizing infections, and affects annually an estimated 12 million people worldwide (Kedzierski 2011). Among diseases of parasitic origin, visceral leishmaniasis is the second leading cause of mortality in humans (Alvar et al. 2012; Bern et al. 2008; Kaye and Scott 2011). The parasite exhibits a digenetic life cycle in which the extracellular promastigotes reside in the gut of the sand fly vector, whereas the intracellular amastigotes inhabit the phagolysosomes of macrophages in the infected mammalian host. Due to the absence of effective vaccines, chemotherapy offers the only avenue of defense against leishmaniasis (Kaye and Scott 2011; Kedzierski et al. 2009; Muller 2007). However, the currently available small arsenal of drugs used to treat leishmaniasis is far from ideal due to a lack of selectivity and emergence of drug resistance (Croft 
et al. 2006; Mishra et al. 2007; Ponte-Sucre et al. 2017; Singh et al. 2012). Thus, the need for a better understanding of parasite biology to develop new therapeutic strategies is urgent.

One pathway that has already been validated as a therapeutic target in the related pathogen, Trypanosoma brucei gambiense, is that for polyamine biosynthesis (Babokhov et al. 2013; Bacchi and McCann 1987; Burri and Brun 2003; Docampo and Moreno 2003; Fairlamb 2003). D,L- $\alpha-$ Difluoromethylornithine (DFMO) is a suicide inhibitor of ornithine decarboxylase (ODC), the enzyme that catalyzes putrescine biosynthesis, and shows remarkable therapeutic efficacy in treating African sleeping sickness caused by T. brucei gambiense (Babokhov et al. 2013; Bacchi and McCann 1987; Burri and Brun 2003; Docampo and Moreno 2003; Fairlamb 2003). DFMO is also active against other protozoan parasites in vitro, including Plasmodia and Giardia (Bitonti et al. 1987; Gillin et al. 1984) and is effective against Leishmania in vitro and in murine and hamster infectivity models (Boitz et al. 2009; Gradoni et al. 1989; Kaur et al. 1986; Mukhopadhyay and Madhubala 1993; Olenyik et al. 2011).

The polyamines putrescine, spermidine, and spermine are ubiquitous and essential cations that play critical roles in proliferation and survival, although their exact functions are still unclear (Igarashi and Kashiwagi 2010; Lenis et al. 2017; Miller-Fleming et al. 2015; Minois et al. 2011; Pegg 2016). Whether the three polyamines have different or overlapping functions and whether all three polyamines are essential remains unknown (Bachrach et al. 2001; Igarashi and Kashiwagi 2010). These analyses are particularly difficult due to the inter-conversion pathways that exist in mammalian cells (Seiler 2004; Seiler et al. 1981; Wang and Casero 2006). As putrescine levels are typically low in mammalian cells, this polyamine is often considered to be merely a precursor for spermidine and spermine formation (Battaglia et al. 2014; Casero and Marton 2007; Kahana 2018; Murray-Stewart et al. 2016; Tavladoraki et al. 2012). It is intriguing that while spermidine and spermine predominate in metazoans, putrescine and spermidine are more abundant in rapidly proliferating prokaryotes and unicellular eukaryotes (Igarashi and Kashiwagi 2010). In contrast to the plethora of studies on the functions of polyamines in mammalian cells, little is known about the functions of polyamines in protozoan parasites, although recent studies have highlighted the importance of the polyamine biosynthetic pathway as a potential therapeutic target (Birkholtz et al. 2011; Bisceglia et al. 2018; Heby et al. 2003, 2007; Ilari et al. 2015, 2017; Phillips 2018; Roberts and Ullman 2017).

The polyamine biosynthetic pathway in Leishmania consists of four enzymes: arginase (ARG), ODC, spermidine synthase (SPDSYN), and $S$-adenosylmethionine decarboxylase (ADOMETDC) (Fig. 1). ARG, the first and committed step in polyamine biosynthesis, converts arginine to ornithine, which is subsequently metabolized to the diamine putrescine by the catalytic action of ODC. SPDSYN then generates spermidine via the addition of an aminopropyl group donated from decarboxylated $S$-adenosylmethionine. Spermine, a prevalent polyamine of higher eukaryotes, is neither synthesized nor utilized by Leishmania (Jiang et al. 1999) and no spermine synthase homolog can be found in Leishmania genomes (El-Sayed et al. 2005). Unique to trypanosomatids, which include T. brucei, T. cruzi, and Leishmania spp., is the conjugation of spermidine and glutathione to form trypanothione. This metabolite is essential to combat oxidative stress in parasites (Colotti and Ilari 2011; Ilari et al. 2017; Krauth-Siegel et al. 2003, 2007; Krauth-Siegel and Comini 2008; Krauth-Siegel and Inhoff 2003; Manta et al. 2013, 2018). In Leishmania, the enzyme trypanothione synthetase-amidase (TRYS) catalyzes the reversible formation of trypanothione (Fyfe et al. 2008). Spermidine is also used for the hypusination and activation of eukaryotic initiation factor 5A (eIF5A). The enzymes deoxyhypusine synthase (DHS) and deoxyhypusine hydroxylase (DOOH) are responsible for this reaction that has been found to be essential in Leishmania (Chawla et al. 2010, 2012) and the mammalian host (Park and Wolff 2018).

ARG, ODC, SPDSYN, and ADOMETDC have all been validated as indispensable for the promastigote form of Leishmania donovani, as gene knockouts of each enzyme confer polyamine auxotrophy to the mutants, which can only be grown in the presence of appropriate polyamine supplementation (Jiang et al. 1999; Roberts 2013; Roberts et al. 2001, 2002, 2004). Studies with $\Delta$ arg knockout mutants in several Leishmania species established that the sole essential role of ornithine is as a precursor for polyamine formation (Boitz et al. 2016; da Silva and Floeter-Winter 2014; da Silva et al. 2012; Reguera et al. 2009; Roberts et al. 2004). In contrast, the role of putrescine is more controversial. Although spermidine is the final polyamine of the pathway in Leishmania, and some studies suggest that spermidine is indeed the only essential polyamine (Jiang et al. 1999; Reguera et al. 2009), a more recent study shows that putrescine is essential beyond its role as a precursor for spermidine formation (Boitz et al. 2016).

Murine infectivity studies have established an important role for parasite ARG in several cutaneous Leishmania species, L. mexicana, L. major, and L. amazonensis (da Silva et al. 2012; Gaur et al. 2007; Muleme et al. 2009; Reguera et al. 2009), and the visceralizing species $L$. donovani (Boitz et al. 2016). The $\Delta$ arg knockout mutants in these strains exhibit lower infectivity levels than the corresponding wild-type parasites, although they are still able to establish infections (Boitz et al. 2016; da Silva et al. 2012; Gaur et al. 2007; Muleme et al. 2009; Reguera et al. 2009). In contrast, $\Delta o d c$ and $\Delta$ spdsyn gene deletion mutants in L. donovani elicit profound 


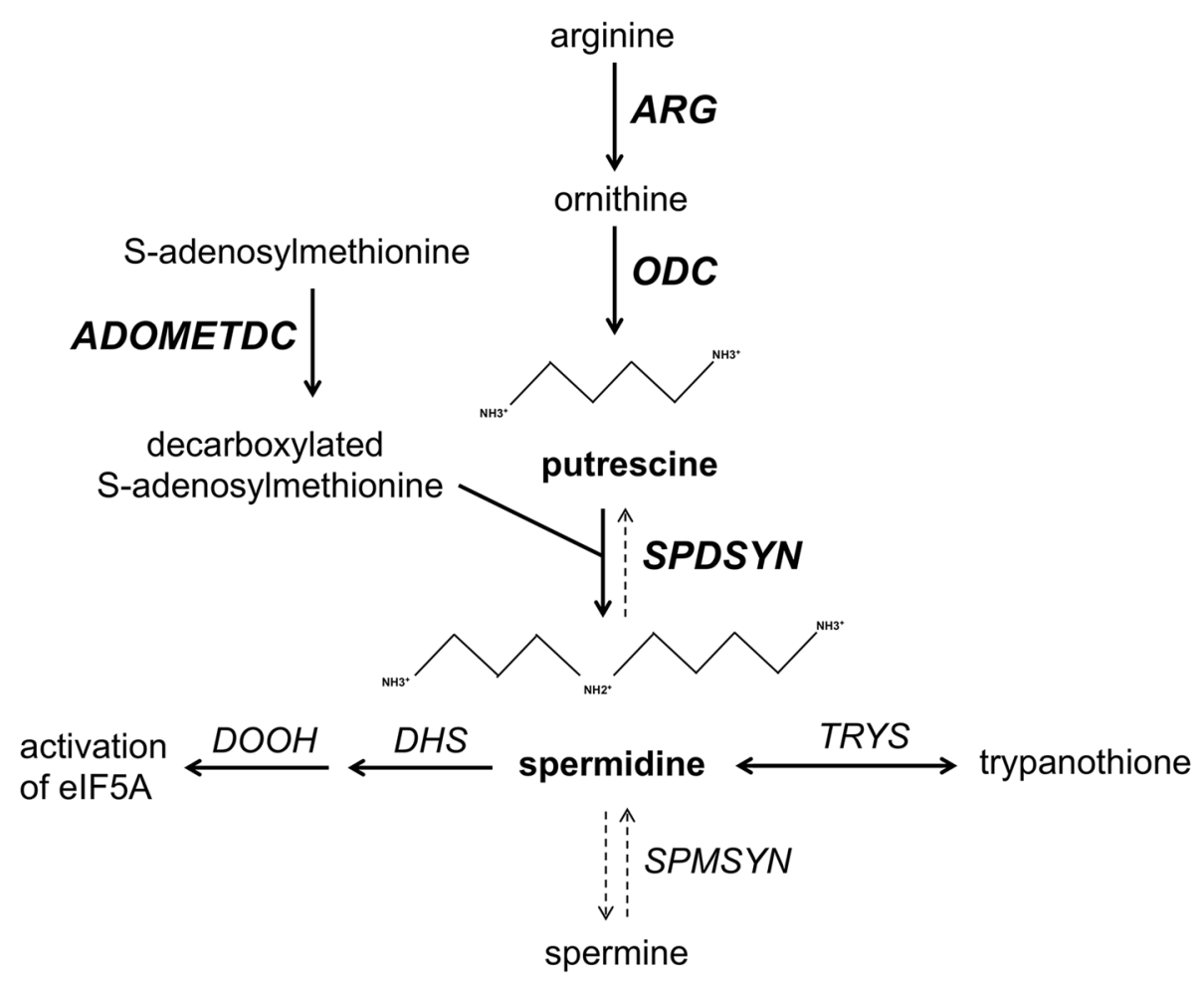

Fig. 1 The polyamine biosynthetic pathway. The polyamine biosynthetic pathway in Leishmania parasites is outlined in bold arrows. The linear conversion of arginine to ornithine, putrescine, and spermidine is catalyzed by arginase (ARG), ornithine decarboxylase (ODC), and spermidine synthase (SPDSYN), respectively. The enzyme $S$-adenosylmethionine decarboxylase (ADOMETDC) forms decarboxylated $S$-adenosylmethionine, the aminopropyl-group donor for the formation of spermidine. The two polyamines found in Leishmania parasites, putrescine and spermidine, are bolded. Unique to trypa-

reductions in parasite loads in infected mouse organs, with the $\Delta$ odc deletion having the most striking effect (Boitz et al. 2009; Gilroy et al. 2011). These studies established the importance of polyamine biosynthesis in intracellular Leishmania parasites.

In this study, we have examined the consequences of polyamine depletion in $L$. donovani $\Delta o d c$ and $\Delta s p d s y n$ gene deletion mutants to better understand the functions of polyamines in Leishmania. Putrescine was not only essential as a precursor for spermidine formation but also critical for parasite proliferation, morphology, and viability. Furthermore, while the absence of both polyamines caused imminent cell death, the presence of either putrescine or spermidine alone, allowed parasites to arrest in a quiescent-like state for several weeks. nosomatids is the formation of trypanothione; in Leishmania this reversible reaction is catalyzed by trypanothione synthetase-amidase (TRYS). The modification and activation of eukaryotic initiation factor 5A (eIF5A) by deoxyhypusine synthase (DHS) and deoxyhypusine hydroxylase (DOOH) occurs in both Leishmania parasites and the human host. Denoted in dashed arrows are the spermine synthase (SPMSYN) reaction and the simplified back-conversion pathway that occur in the mammalian host but are not present in Leishmania parasites

\section{Materials and methods}

\section{Materials}

Dulbecco's Modified Eagle's Medium, chicken serum, and phleomycin were procured from Thermo Fisher Scientific (Waltham, MA, USA). Resazurin, putrescine, and spermidine were purchased from VWR International (Radnor, PA, USA). Dansyl chloride was obtained from SigmaAldrich (Burlington, MA, USA), and the Muse Count and Viability reagent was purchased from Millipore (Burlington, MA, USA). 


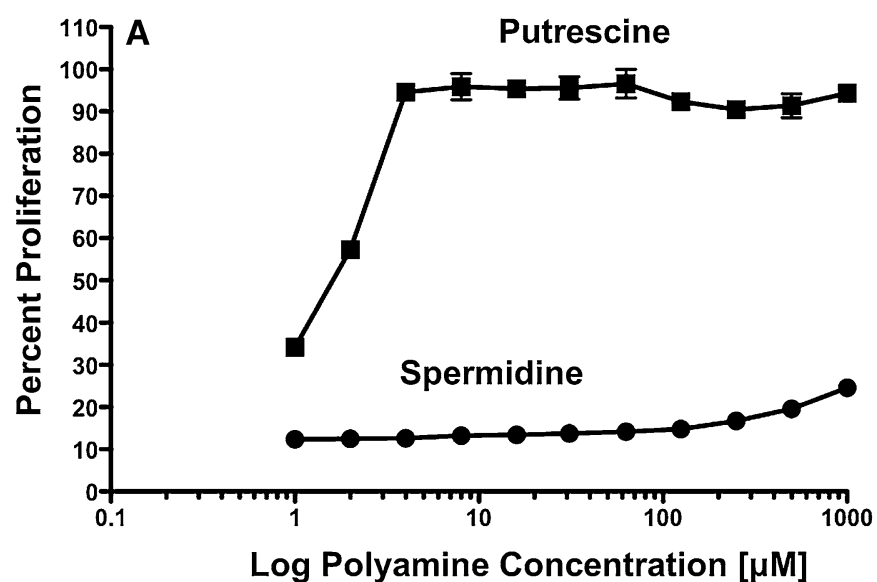

Fig. 2 Putrescine and spermidine concentrations required to rescue mutant parasites. The $\Delta o d c$ (a) and $\Delta s p d s y n$ (b) promastigotes were incubated in serial dilutions of $1000 \mu \mathrm{M}$ putrescine (black squares) or $1000 \mu \mathrm{M}$ spermidine (black circles). Viability was evaluated after

\section{Cell culture and cell lines}

All genetically manipulated parasites were derived from the wild-type LdBob strain of L. donovani (Goyard et al. 2003) that was originally obtained from Dr. Stephen M. Beverley (Washington University, St. Louis, MO, USA). The $\Delta o d c$ and $\Delta$ spdsyn mutants were previously generated by targeted gene replacement techniques (Boitz et al. 2009; Gilroy et al. 2011). Promastigote parasites were incubated at $27^{\circ} \mathrm{C}$ in a completely defined Dulbecco's Modified Eagle-based culture medium especially designed for the cultivation of Leishmania promastigotes, where fetal bovine serum was replaced with chicken serum to avoid polyamine oxidase-mediated toxicity (DME-L CS) (Iovannisci and Ullman 1983; Kaur et al. 1986; Roberts et al. 2001). The $\Delta$ spdsyn cell line was maintained in $100 \mu \mathrm{M}$ spermidine and the $\Delta o d c$ cell line was routinely grown in the presence of $100 \mu \mathrm{M}$ putrescine, unless otherwise specified. Prior to performing the experiments described in this paper, the phenotype of the $\Delta o d c$ and $\Delta$ spdsyn gene deletion mutants was verified by western blot analysis. Blots were probed with polyclonal rabbit antibodies raised against ODC and SPDSYN (Jiang et al. 1999; Roberts et al. 2001) and tubulin as a loading control, and the absence of ODC in the $\Delta o d c$ cell lines and SPDSYN in the $\Delta s p d s y n$ parasites was confirmed (Supplemental Fig. 1).

\section{Assessment of growth phenotypes}

Wild-type, $\Delta o d c$, and $\Delta s p d s y n$ parasites were allowed to reach stationary phase in media supplemented with no polyamines (wild-type), with $100 \mu \mathrm{M}$ putrescine $(\Delta o d c)$, or with $100 \mu \mathrm{M}$ spermidine $(\Delta$ spdsyn). Parasites were then harvested and washed three times in phosphate buffered saline (PBS),

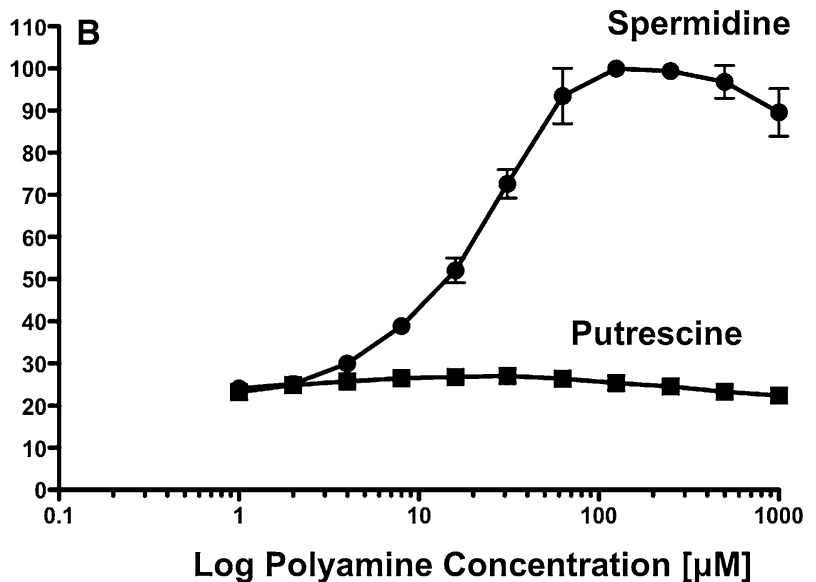

5 days by measuring the conversion of resazurin to resorufin. Data are from two biological replicates $(n=2)$ with error bars representing standard deviations. The experiment was repeated two more times with essentially the same outcome

counted on a hemocytometer and seeded at defined cell numbers for all growth assays. To determine $50 \%$ effective concentration $\left(\mathrm{EC}_{50}\right)$ values and concentrations of putrescine and spermidine required for optimal growth, $\Delta o d c$ and $\Delta$ spdsyn parasites were seeded in a volume of $100 \mu \mathrm{l}$ in 96-well plates at a density of $5 \times 10^{4} / 100 \mu \mathrm{l}$ in serial dilutions of $1000 \mu \mathrm{M}$ putrescine or $1000 \mu \mathrm{M}$ spermidine. After 5 days, $15 \mu \mathrm{l}$ of $250 \mu \mathrm{M}$ resazurin was added to each well, and plates were incubated for an additional $4 \mathrm{~h}$. To assess cellular proliferation, conversion of resazurin to resorufin was evaluated on a BioTek Synergy plate reader by monitoring fluorescence $\left(579_{\mathrm{Ex}} / 584_{\mathrm{Em}}\right)$. Graphs were prepared using GraphPad Prism version 6.0f for Mac. Data shown in Fig. 2 are from biological duplicates $(n=2)$. The experiment was repeated two more times with essentially the same outcome. To determine daily parasite growth rates, wild-type, $\Delta o d c$, and $\Delta$ spdsyn parasites were seeded in a volume of $10 \mathrm{ml}$ at a density of $5 \times 10^{5} / \mathrm{ml}$ and counted in a hemocytometer daily for 7 days. The $\Delta o d c$ and $\Delta s p d s y n$ parasites were incubated in media containing no polyamines, $100 \mu \mathrm{M}$ putrescine, $100 \mu \mathrm{M}, 500 \mu \mathrm{M}$, or $1000 \mu \mathrm{M}$ spermidine as indicated. Graphs were prepared using Microsoft Excel. Data displayed in Fig. 3 are from biological duplicates $(n=2)$. The experiment was repeated two more times with essentially the same outcome.

\section{Polyamine pool analysis}

Wild-type, $\Delta o d c$, and $\Delta s p d s y n$ parasites were allowed to reach stationary phase in media supplemented with no polyamines (wild-type), with $100 \mu \mathrm{M}$ putrescine $(\Delta o d c)$, or with $100 \mu \mathrm{M}$ spermidine $(\Delta s p d s y n)$. Parasites were then harvested and washed three times in PBS. Wild-type parasites were 


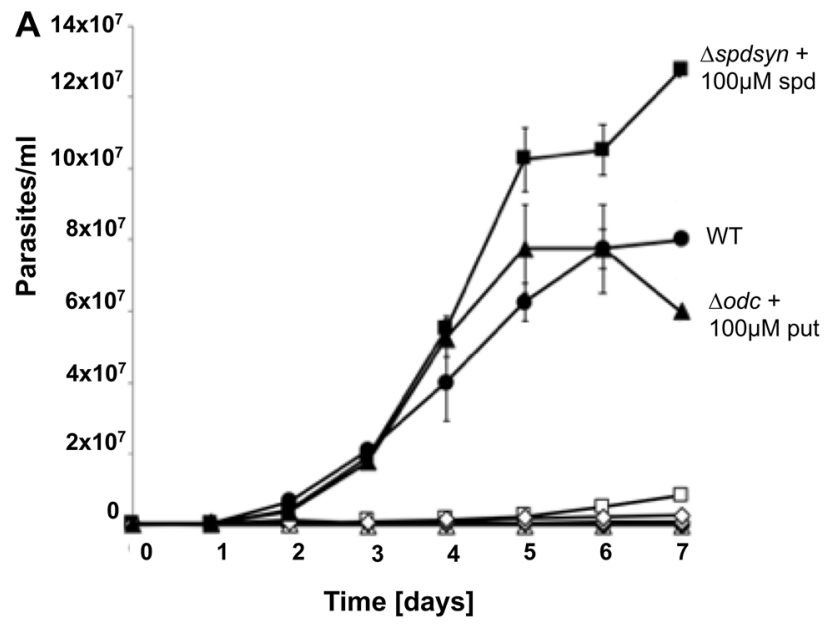

Fig. 3 Proliferation of wild-type and mutant parasites in supplemented or un-supplemented media. Parasites were seeded at $5 \times 10^{5}$ parasites $/ \mathrm{ml}$ and proliferation was evaluated daily by counting parasite numbers on a hemocytometer. a Depicts growth of all cell lines, while $\mathbf{b}$ focuses on growth of starved parasites to allow a better comparison of low cellular proliferation rates. Wild-type parasites were incubated in un-supplemented media (black circles), $\Delta o d c$ parasites were incubated in un-supplemented media (white triangles) or sup-

then incubated in media without polyamines, $\Delta o d c$ parasites in the absence of polyamines, or in the presence of $100 \mu \mathrm{M}$ putrescine, or $100 \mu \mathrm{M}, 500 \mu \mathrm{M}$, or $1000 \mu \mathrm{M}$ spermidine, and $\Delta$ spdsyn parasites in the absence of polyamines or in the presence of $100 \mu \mathrm{M}$ spermidine. Parasites $\left(1 \times 10^{7}\right)$ were harvested after 3 days, washed three times in PBS, and extracted for polyamine pool determination with $10 \%$ trichloroacetic acid (TCA) as described previously (Jiang et al. 1999; Shim and Fairlamb 1988). An internal standard, 1,7-diaminoheptane, was added to the polyamine-TCA solutions for further processing. Samples were extracted in ethyl acetate and dried on a Speed Vac concentrator. The samples were derivatized with a solution of dansyl chloride (fluorescent label) and proline was added to scavenge the excess dansyl chloride. The derivatized polyamines were recovered with two ethyl acetate extractions, the organic layers were pooled and dried, and samples were dissolved in $200 \mu \mathrm{l}$ of $95 \%$ methanol $/ 5 \%$ acetic acid. The polyamines were separated by highperformance liquid chromatography (flow rate $=1 \mathrm{ml} / \mathrm{min}$ ) on a Shimadzu Prominence system equipped with a Phenomenex Kinetix $\mathrm{C}_{18} \mathrm{XB}$ reversed-phase column $(100 \times 4.6 \mathrm{~mm}$; $5 \mu \mathrm{m} ; 100 \AA$ ). Gradient elution (45\% B-80\% B from 0 to $14 \mathrm{~min} ; 80 \%$ B from 14 to $15 \mathrm{~min}$ ) with solvent A (10 mM sodium phosphate; $\mathrm{pH}=7.2$ ) and solvent $\mathrm{B}$ (acetonitrile) was used to separate putrescine (retention time $\approx 8.6 \mathrm{~min}$ ), spermidine (retention time $\approx 13.9 \mathrm{~min}$ ), and the internal standard 1,7-diaminoheptane (retention time $\approx 11.1 \mathrm{~min}$ ). Fluorescence was measured on a Shimadzu RF-535 detector $\left(340_{\mathrm{Ex}} / 515_{\mathrm{EM}}\right)$. LabSolutions version 5.73 by Shimadzu

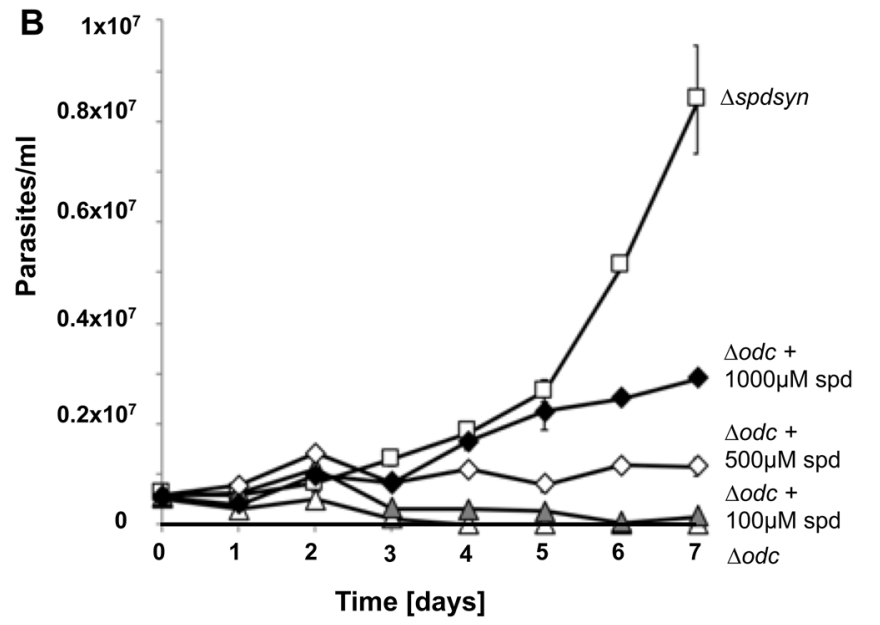

plemented with $100 \mu \mathrm{M}$ spermidine (gray triangles), $500 \mu \mathrm{M}$ spermidine (white diamonds), or $1000 \mu \mathrm{M}$ spermidine (black diamonds), or supplemented with $100 \mu \mathrm{M}$ putrescine (black triangle), and $\Delta$ spdsyn parasites were incubated un-supplemented media (white squares) or supplemented with $100 \mu \mathrm{M}$ spermidine (black squares). Data are from two biological duplicates $(n=2)$ with error bars representing standard deviations. The experiment was repeated two more times with essentially the same outcome

Corporation (Kyoto, Japan) software was used to calculate peak areas. A comparison of peak areas from samples to standard curves of putrescine and spermidine, which contained the internal standard 1,7-diaminoheptane, was used to quantify the individual polyamines. The experiments were conducted three times with biological triplicates for each experiment and the data (total $n=9$ per cell line and condition) are shown in Fig. 4. The unpaired $t$ test was used to evaluate statistical significance.

\section{Microscopy}

Wild-type, $\Delta o d c$, and $\Delta s p d s y n$ parasites were allowed to reach stationary phase in media supplemented with no polyamines (wild-type), with $100 \mu \mathrm{M}$ putrescine $(\Delta o d c)$, or with $100 \mu \mathrm{M}$ spermidine $(\Delta s p d s y n)$. Parasites were then harvested, washed three times in PBS, and seeded in media with or without supplementation with polyamines. After 3 days, $500 \mu \mathrm{l}$ parasites were harvested, washed once in PBS, and $20 \mu \mathrm{l}$ samples were placed on poly-L-lysine-coated slides. Coverslips were applied without sealing. Bright-field images were taken on an AMG Evos XL at $40 \times$ magnification within 10 min of adding samples to the slides. The experiment was repeated more than three times and visual observations were consistent. Cell body lengths, excluding the flagellum, were measured using an Olympus BX41 microscope at $40 \times$ magnification utilizing the Neurolucida software (MBF Biosciences). Three independent experiments were performed and 50-100 cells for each parasite 

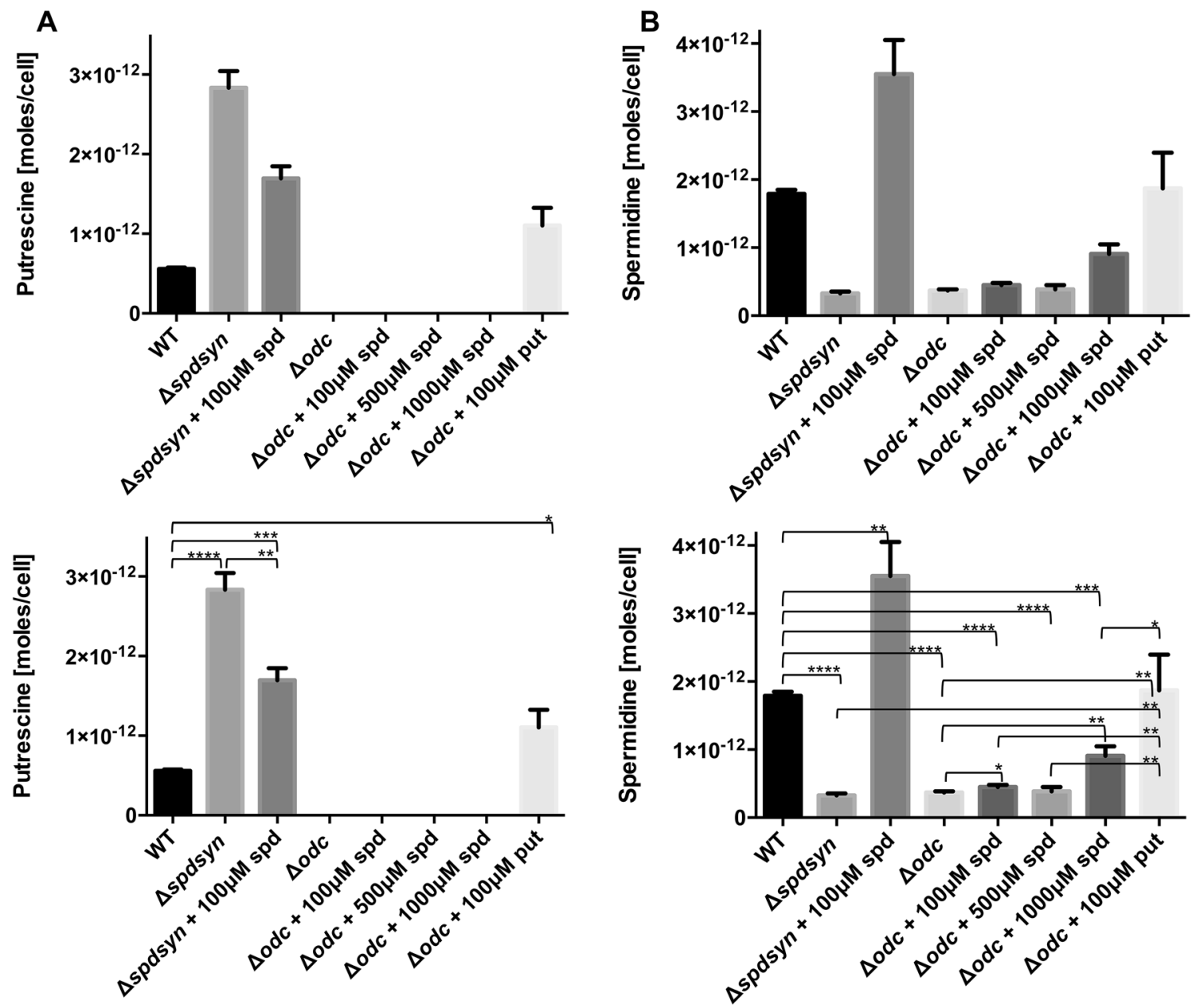

Fig. 4 Polyamine levels in wild-type and mutant parasites in supplemented or un-supplemented media. Wild-type parasites were incubated in media without polyamines, $\Delta o d c$ parasites were incubated in the absence of polyamines, or in the presence of $100 \mu \mathrm{M}$ putrescine, $100 \mu \mathrm{M}, 500 \mu \mathrm{M}$, or $1000 \mu \mathrm{M}$ spermidine, and $\Delta$ spdsyn parasites were incubated in the absence of polyamines or in the presence of $100 \mu \mathrm{M}$ spermidine. Parasites were harvested after 3 days and polyamine levels were measured in wild-type and mutant parasites. a Shows putrescine levels and $\mathbf{b}$ depicts spermidine levels. The experi-

strain and condition were measured in each experiment, with a total of 200-250 cells measured for each parasite strain and condition. The unpaired $T$ test was performed using GraphPad software for statistical analysis.

\section{Viability assay}

Wild-type, $\Delta o d c$, and $\Delta s p d s y n$ parasites were allowed to reach stationary phase in media supplemented with no polyamines (wild-type), with $100 \mu \mathrm{M}$ putrescine $(\Delta o d c)$, or with $100 \mu \mathrm{M}$ spermidine $(\Delta s p d s y n)$. Parasites were then harvested, washed three times in PBS, and seeded in media

ments were conducted three individual times with three biological replicates for each experiment and the data (total $n=9$ per cell line and condition) are shown with error bars representing standard deviations. The unpaired $T$ test was performed using GraphPad software for statistical analysis, which is shown in the lower panels. A $p$ value of $<0.05$ is denoted with one asterisk, a $p$ value of $<0.01$ with two asterisks, a $p$ value of $<0.001$ with three asterisks, and a $p$ value of $<0.0001$ with four asterisks

with or without supplementation with polyamines. All cell lines were seeded at $3 \times 10^{5} / \mathrm{ml}$ and samples were taken daily. Viability was assessed on a Muse ${ }^{\circledR}$ Cell Analyzer (Millipore Sigma) using the Muse ${ }^{\mathrm{TM}}$ Count and Viability reagent according to the manufacturer's manual. Briefly, Count and Viability reagent was added to each microcentrifuge containing parasite samples and incubated for $5 \mathrm{~min}$ at room temperature before inserting into the Muse ${ }^{\circledR}$ Cell Analyzer. Graphs were prepared using GraphPad. Data from three biological replicates $(n=3)$ are displayed in Fig. 6. The experiment was repeated two more times with essentially the same outcome. 


\section{Rescue assay}

Parasite survival was determined with a long-term rescue assay. Wild-type, $\Delta o d c$, and $\Delta s p d s y n$ parasites were allowed to reach stationary phase in media supplemented with no polyamines (wild-type), with $100 \mu \mathrm{M}$ putrescine $(\Delta o d c)$, or with $100 \mu \mathrm{M}$ spermidine $(\Delta s p d s y n)$. Parasites were then harvested and washed three times in PBS. The $\Delta o d c$ mutants were seeded at $5 \times 10^{6}$ cells $/ \mathrm{ml}$ in $10 \mathrm{ml}$ media without polyamine supplementation or in media containing $100 \mu \mathrm{M}$ spermidine. The $\Delta$ spdsyn mutants were seeded at $1 \times 10^{6}$ cells $/ \mathrm{ml}$ in $10 \mathrm{ml}$ media without polyamine supplementation. Multiple flasks were set up for each cell line and condition. Over the course of 18 weeks, weekly $500 \mu \mathrm{l}$ aliquots were taken from two flasks per cell line and condition, spun down, and pellets were resuspended in $1 \mathrm{ml}$ media containing either $100 \mu \mathrm{M}$ putrescine $(\Delta o d c)$ or $100 \mu \mathrm{M}$ spermidine $(\Delta$ spdsyn). The $1 \mathrm{ml}$ parasite cultures were transferred into 24-well plates. One or two weeks after the rescue attempt, parasite survival was assessed under the microscope. Samples that showed healthy and motile parasites were deemed alive (rescued), while samples that showed non-motile forms were deemed dead. The experiment was repeated six times for $\Delta o d c$ and $\Delta$ spdsyn mutants incubated in media without polyamine supplementation and three times for $\Delta o d c$ parasites incubated in media with $100 \mu \mathrm{M}$ spermidine. Each time, the rescue attempts were performed in duplicates.

\section{Results}

\section{Polyamine requirements of gene deletion mutants}

To establish $\mathrm{EC}_{50}$ values for putrescine and spermidine, and determine concentrations necessary for optimal growth of $\Delta o d c$ and $\Delta s p d s y n$ parasites, mutants were incubated in serial dilutions of $1000 \mu \mathrm{M}$ putrescine or $1000 \mu \mathrm{M}$ spermidine. Cell growth was assessed by measuring the conversion of resazurin to resorufin after 5 days (Fig. 2). The $\Delta o d c$ parasites required the addition of $5.80 \pm 1.56 \mu \mathrm{M}$ putrescine to the media for optimal growth, with an $\mathrm{EC}_{50}$ of $1.88 \pm 0.54 \mu \mathrm{M}$ (average of three experiments performed in duplicates). The downstream metabolite spermidine was not able to restore growth of $\Delta o d c$ parasites at concentrations up to $1000 \mu \mathrm{M}$. The $\Delta s p d s y n$ parasites depended on $55.00 \pm 7.07 \mu \mathrm{M}$ spermidine supplementation for ideal growth, with an $\mathrm{EC}_{50}$ of $11.5 \pm 9.19 \mu \mathrm{M}$ (average of three experiments performed in duplicate). Supplementation of $\Delta$ spdsyn parasites with putrescine, the substrate for the spermidine synthase reaction, did not rescue the growth defect of the $\Delta s p d s y n$ mutants. As $\Delta s p d s y n$ parasites are able to synthesize putrescine, the addition of putrescine was not expected to have an effect on growth.

\section{Proliferation of polyamine-starved parasites}

Proliferation of $\Delta o d c$ and $\Delta s p d s y n$ parasites incubated in polyamine-free or supplemented media was compared by counting cell numbers over the course of 7 days (Fig. 3). Wild-type parasites, $\Delta o d c$ mutants supplemented with $100 \mu \mathrm{M}$ putrescine, and $\Delta$ spdsyn mutants supplemented with $100 \mu \mathrm{M}$ spermidine proliferated optimally over the course of the experiment (Fig. 3a). It should be noted that the supplemented $\Delta$ spdsyn mutants showed slightly higher growth rates than the wild-type or supplemented $\Delta o d c$ mutants in Fig. 3a. However, growth curves of wild-type parasites and supplemented mutants were repeated numerous times and within the range of normal fluctuations these three cell lines proliferated equally well, which has also been reported previously (Boitz et al. 2009; Gilroy et al. 2011). In contrast, the $\Delta o d c$ mutants grown in polyamine-free media arrested cell division immediately (Fig. 3b). To investigate if this severe growth defect was due to the depletion of putrescine alone or both putrescine and spermidine, the $\Delta o d c$ mutants were incubated in the presence of $100 \mu \mathrm{M}, 500 \mu \mathrm{M}$, or $1000 \mu \mathrm{M}$ spermidine. Some improvement in cell growth was observed in $\Delta$ odc parasites in media supplemented with $500 \mu \mathrm{M}$ or $1000 \mu \mathrm{M}$ spermidine; however, it did not reach the level of growth observed in $\Delta$ spdsyn mutants (Fig. 3b). In comparison with the $\Delta o d c$ mutants, the $\Delta s p d s y n$ parasites incubated in polyamine-free media exhibited an intermediate growth phenotype (Fig. $3 b$ ). The $\Delta s p d s y n$ parasites were initially seeded at $5 \times 10^{5}$ parasites $/ \mathrm{ml}$ and reached a density of about $1 \times 10^{7}$ parasites $/ \mathrm{ml}$ after 7 days, indicating that cells divided four to five times.

\section{Correlation of growth phenotypes with intracellular putrescine and spermidine levels}

To correlate the observed growth deficits with intracellular putrescine and spermidine levels, polyamine pools were measured in wild-type and mutant parasites. Wild-type cells were incubated in media without supplementation, and the $\Delta o d c$ and $\Delta s p d s y n$ gene deletion mutants were incubated in media with or without polyamine supplementations for 3 days before parasites were harvested for the analysis of intracellular polyamine content.

Intracellular putrescine levels were below the detection level in $\Delta o d c$ parasites incubated in polyamine-free media or in media containing various concentrations of spermidine (Fig. 4). When the $\Delta o d c$ mutants were incubated in media supplemented with $100 \mu \mathrm{M}$ putrescine, the intracellular putrescine levels were about twofold higher than in wild-type parasites, suggesting that a robust uptake of putrescine occurred in these cells to counter the inability to synthesize putrescine. Putrescine levels were about threeand fivefold higher in $\Delta$ spdsyn mutants incubated in media 
Fig. 5 Morphology of wild-type and mutant parasites in supplemented or un-supplemented media. Bright-field images of parasites were taken on an Evos microscope after 3 days of incubation. Wild-type parasites were grown in media without polyamine supplementation, $\Delta$ spdsyn were grown without polyamines or in the presence of $100 \mu \mathrm{M}$ spermidine, and $\Delta o d c$ with incubated in the presence of $100 \mu \mathrm{M}$ putrescine, $100 \mu \mathrm{M}$ spermidine or no polyamine supplementation. The experiment was repeated more than three times and visual observations were consistent

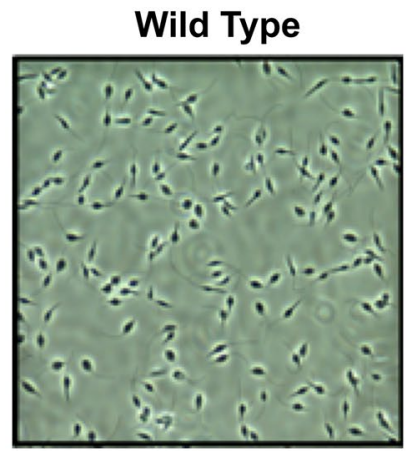

$\Delta$ spdsyn + spd

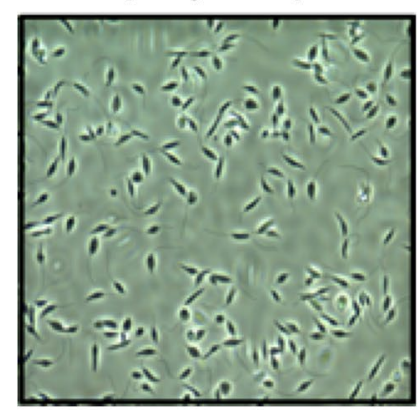

$\Delta o d c+$ spd
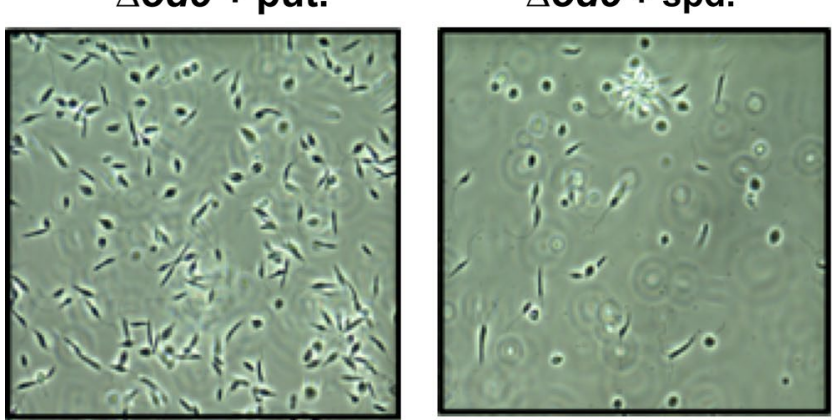

$\Delta$ spdsyn

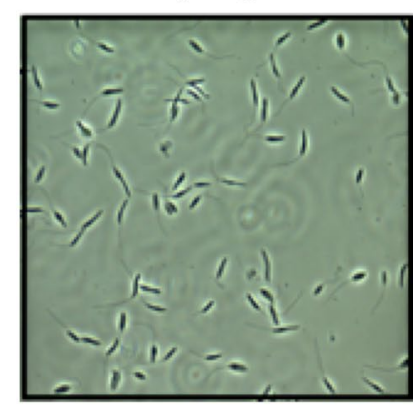

$\Delta o d c$

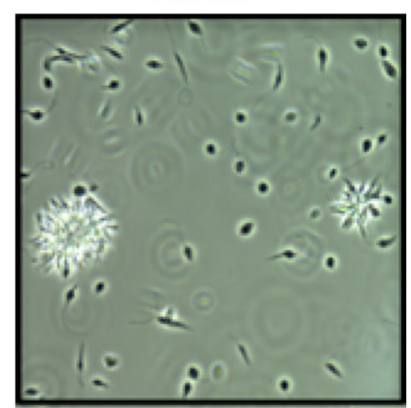

with or without spermidine, respectively, than in wild-type parasites. This observation can likely be ascribed to an accumulation of putrescine due to the inability of $\Delta s p d s y n$ mutants to convert putrescine to spermidine.

Intracellular spermidine levels in the $\Delta o d c$ and $\Delta s p d s y n$ parasites incubated in spermidine-free media were considerably lower than in wild-type parasites. However, in contrast to the profound depletion of putrescine levels in $\Delta o d c$ mutants (below detection level), spermidine levels in both $\Delta o d c$ and $\Delta s p d s y n$ parasites were only about fivefold lower than in wild-type parasites. Supplementation of $\Delta$ spdsyn mutants with $100 \mu \mathrm{M}$ spermidine resulted in a twofold increase of spermidine levels compared to wild-type parasites, suggesting robust uptake to counter the inability to synthesize spermidine. In contrast, supplementation of $\Delta o d c$ parasites with 100,500 , or $1000 \mu \mathrm{M}$ spermidine did not restore spermidine levels to that of wild-type parasites. In fact, only supplementation of $\Delta o d c$ parasites with $1000 \mu \mathrm{M}$ spermidine caused a modest increase of spermidine levels compared to those of $\Delta o d c$ parasites incubated in polyamine-free media.

Taken together, the analysis of intracellular polyamine levels revealed that both mutant cell lines, $\Delta o d c$ and $\Delta s p d s y n$, contained low levels of intracellular spermidine but only the $\Delta s p d s y n$ mutants contained putrescine. Thus, the immediate cessation of cellular proliferation and other phenotypic observations in the starved $\Delta o d c$ cell line (described below) correlated with the loss of putrescine.

\section{Morphology of polyamine-starved parasites}

The effect of polyamine deficiencies on parasite morphology was assessed. The $\Delta o d c$ mutants incubated in polyaminefree or spermidine-supplemented media exhibited a more rounded morphology (Fig. 5), were less motile (data not shown) and more prone to form clusters compared to wildtype or putrescine-supplemented $\Delta o d c$ parasites. In contrast, $\Delta$ spdsyn parasites incubated in media without spermidine showed cell shapes and motility similar to wild-type or supplemented $\Delta s p d s y n$ mutants (Fig. 5). Cell body lengths (without flagella) were measured and compared for the different cell lines and supplement conditions after 3 days of starvation. The body length of putrescine-depleted $\Delta o d c$ parasites averaged $6.27 \pm 1.17 \mu \mathrm{m}$, while putrescine-supplemented $\Delta o d c$ and wild-type parasites exhibited lengths of $11.88 \pm 1.43 \mu \mathrm{m}$ and $14.41 \pm 0.17 \mu \mathrm{m}$, respectively (Table 1). In contrast, the body length of spermidine-depleted $\Delta s p d s y n$ parasites, $12.51 \pm 1.18 \mu \mathrm{m}$, was similar to that of spermidine-supplemented $\Delta$ spdsyn, $12.22 \pm 0.63 \mu \mathrm{m}$, and wild-type parasites, $14.41 \pm 0.17 \mu \mathrm{m}$. Statistical analysis (unpaired student $t$ test) confirmed that the differences between the body lengths of the putrescine-depleted $\Delta o d c$ parasites and the putrescine-supplemented $\Delta o d c$ parasites or wild-type parasites were statistically significant.

\section{Viability of polyamine-starved parasites}

The putrescine-starved $\Delta o d c$ parasites ceased proliferation immediately (Fig. 3), demonstrated a rounded morphology 
Table 1 Body length of wild-type and mutant parasites

\begin{tabular}{lcl}
\hline Cell line and supplement & $\begin{array}{l}\text { Cell length in } \mu \mathrm{m} \\
\text { Mean (aver- } \\
\text { age) } \pm \text { standard } \\
\text { deviation }\end{array}$ & $\begin{array}{l}\text { Total number of } \\
\text { cells measured }\end{array}$ \\
\hline $\mathrm{WT}$ & $14.41 \pm 0.17$ & 200 \\
$\Delta o d c+100 \mu \mathrm{M}$ putrescine & $11.88 \pm 1.43$ & 200 \\
$\Delta o d c$ & $6.27 \pm 1.17$ & 250 \\
$\Delta o d c+100 \mu \mathrm{M}$ spermidine & $7.24 \pm 0.72$ & 200 \\
$\Delta s p d s y n+100 \mu \mathrm{M}$ spermidine & $12.22 \pm 0.63$ & 200 \\
$\Delta s p d s y n$ & $12.51 \pm 1.18$ & 250 \\
\hline
\end{tabular}

Wild-type parasites were grown in media without polyamine supplementation, $\Delta$ spdsyn parasites were grown without polyamines or in the presence of $100 \mu \mathrm{M}$ spermidine, and $\Delta o d c$ mutants were incubated in the presence of $100 \mu \mathrm{M}$ putrescine, $100 \mu \mathrm{M}$ spermidine or no polyamine supplementation. Parasite body length was measured after 3 days of starvation as described in "Materials and methods". The table represents data from three independent experiments, and 50-100 cells for each parasite strain and condition were measured in each experiment. Statistical analysis using the unpaired $T$ test revealed significant differences $(p$ value $<0.001$ ) for $\Delta o d c$ mutants incubated with or without spermidine when compared to the size of wild-type parasites or $\Delta o d c$ mutants incubated in the presence of putrescine
Wild-type parasites, $\Delta$ spdsyn parasites supplemented with spermidine, and $\Delta o d c$ parasites supplemented with putrescine showed a high percentage of viable parasites, 90\%, up to day 4 or 5 (Fig. 6). Once the culture reached stationary phase and a high density, viability plummeted rapidly (Fig. 6). The $\Delta$ spdsyn mutants grown in the absence of polyamines sustained a high viability of above $70 \%$ even after 8 days of starvation, while the $\Delta o d c$ parasites with or without supplementation of spermidine showed a gradual decline in viability, starting by day 2 or 3 , and reached a low $45 \%$ ( $\Delta o d c$ with spermidine) and $30 \%(\Delta o d c)$ viability by day 8 (Fig. 6). The addition of $100 \mu \mathrm{M}$ spermidine improved viability of the $\Delta o d c$ parasites slightly. This analysis demonstrates that the putrescine-depleted $\Delta o d c$ parasites not only ceased proliferation, they also showed a steady decrease of cell viability under polyamine starvation conditions.

\section{Assessment of long-term survival of gene deletion mutants}

Parasite survival was determined over the course of several weeks by a rescue assay. For this assay, parasites were incubated in starvation conditions and samples were taken once

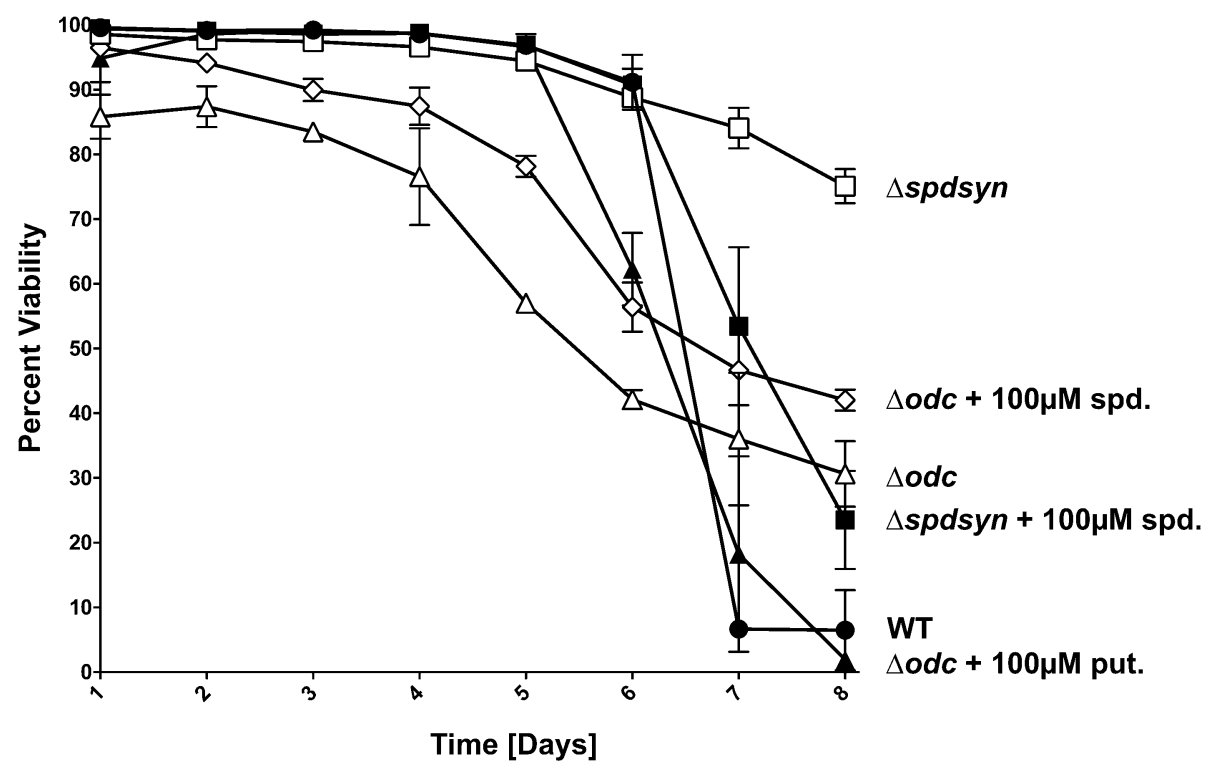

Fig. 6 Viability of wild-type and mutant parasites in supplemented or un-supplemented media. Parasites were seeded at $3 \times 10^{5}$ parasites/ $\mathrm{ml}$ and samples were analyzed by flow cytometry for viability every day for the course of 8 days. Wild-type parasites were incubated in un-supplemented media (black circles), $\Delta o d c$ parasites were incubated in un-supplemented media (white triangles), or supplemented

(Fig. 5 and Table 1), and most parasites appeared to be nonmotile (data not shown). However, as these observations did not discern whether parasites were alive or dead, the percentage of viable parasites was determined by flow cytometry. with $100 \mu \mathrm{M}$ spermidine (white diamonds), or $100 \mu \mathrm{M}$ putrescine (black triangles), and $\Delta$ spdsyn parasites were incubated in un-supplemented media (white squares) or supplemented with $100 \mu \mathrm{M}$ spermidine (black squares). Data are from three biological replicates $(n=3)$ with error bars representing standard deviations. The experiment was repeated two more times with essentially the same outcome

a week. Rescue was attempted by the addition of putrescine or spermidine to the $\Delta o d c$ and $\Delta s p d s y n$ parasite cultures, respectively. The $\Delta o d c$ parasites incubated in polyamine-free 
Table 2 Long-term survival of polyamine-starved parasites

\begin{tabular}{lcl}
\hline Cell line and supplement & $\begin{array}{l}\text { Survival in weeks } \\
\text { Mean (aver- } \\
\text { age) } \pm \text { standard } \\
\text { deviation }\end{array}$ & $\begin{array}{l}\text { Number of } \\
\text { experi- } \\
\text { ments }\end{array}$ \\
\hline$\Delta$ odc & $2.08 \pm 0.51$ & 6 \\
$\Delta s p d s y n$ & $5.92 \pm 0.67$ & 6 \\
$\Delta o d c+100 \mu \mathrm{M}$ spermidine & $14.17 \pm 2.14$ & 3 \\
\hline
\end{tabular}

The $\Delta$ spdsyn and $\Delta o d c$ mutants were incubated in media without polyamine supplementation and $\Delta o d c$ mutants were also incubated in media with $100 \mu \mathrm{M}$ spermidine. Once a week, aliquots were taken and rescued by the addition of putrescine $(\Delta o d c)$ or spermidine $(\Delta s p d s y n)$ as outlined in "Materials and methods". The experiment was repeated six times for $\Delta o d c$ and $\Delta s p d s y n$ mutants incubated in media without polyamine supplementation and three times for $\Delta o d c$ parasites incubated in media with $100 \mu \mathrm{M}$ spermidine. Each time the rescue attempts were performed in duplicates

media survived for only approximately 2 weeks; and could not be rescued by the addition of $100 \mu \mathrm{M}$ putrescine after that time (Table 2). In contrast, the addition of $100 \mu \mathrm{M}$ spermidine allowed $\Delta o d c$ mutants to persist for 14 weeks (Table 2). The $\Delta$ spdsyn parasites incubated in polyaminefree media showed an intermediate survival phenotype and lived for 6 weeks in a quiescent-like state (Table 2).

\section{Discussion}

Our studies demonstrate that both polyamines, putrescine and spermidine, are essential for Leishmania and that putrescine has previously unrecognized vital functions beyond its role as a precursor for spermidine formation. Putrescinedepleted $\Delta o d c$ parasites showed a more profound decrease in proliferation and viability compared to putrescine-rich $\Delta$ spdsyn parasites, pointing towards specific functions of putrescine for these cellular processes.

Spermidine is the final product of the polyamine biosynthetic pathway in Leishmania (Fig. 1) and has been proposed to be the only essential polyamine (Heby et al. 2007; Jiang et al. 1999; Reguera et al. 2009). Indeed, studies have demonstrated that the sole essential function of the amino acid ornithine is as precursor for the polyamine biosynthesis in Leishmania (Boitz et al. 2016; da Silva and Floeter-Winter 2014; da Silva et al. 2012; Reguera et al. 2009; Roberts et al. 2004). Similarly, it has been suggested that putrescine is merely the precursor metabolite for spermidine formation, and supplementation of spermidine was found to be sufficient to at least partially restore growth in L. major $\Delta a r g$ and L. donovani $\Delta$ odc mutants (Jiang et al. 1999; Reguera et al. 2009). However, a recent study found that $L$. donovani $\Delta a r g$ mutants did not proliferate in the presence of spermidine (Boitz et al. 2016) and we now report that supplementation of up to $1000 \mu \mathrm{M}$ spermidine did not rescue $L$. donovani $\Delta o d c$ mutants. Previous observations that spermidine was able to partially restore growth of L. major $\Delta$ arg or $L$. donovani $\Delta$ odc mutants may have been caused by putrescine contaminations of commercially available spermidine. This conjecture is plausible as the $\mathrm{EC}_{50}$ values for putrescine were $7.5 \mu \mathrm{M}$ for L. donovani $\Delta$ arg (Boitz et al. 2016) and $1.88 \mu \mathrm{M}$ for L. donovani $\Delta o d c$ mutants (Fig. 2a), and thus very small amounts of putrescine contamination could be sufficient to allow at least some growth. Our observation that $L$. donovani $\Delta o d c$ mutants showed a slight increase in growth at 500 and $1000 \mu \mathrm{M}$ (Fig. 2a) can possibly also be ascribed to small amounts of putrescine in the spermidine stock solution. The more recent observations that $L$. donovani $\Delta$ arg (Boitz et al. 2016) and $\Delta o d c$ mutants (Fig. 2) require putrescine supplementation is profound as it shifts the current paradigm of the importance of putrescine from being a precursor metabolite to having essential functions on its own. Intriguingly, similar observations have been made in T. brucei, where RNAimediated silencing of ODC could be rescued by supplementation with putrescine but not spermidine and silencing of ODC resulted in a more rapid cell death than silencing of SPDSYN (Xiao et al. 2009). Although this observation was ascribed to the lack of both putrescine and spermidine, it is feasible that putrescine has a uniquely important function for trypanosomatids.

The experiments presented here reveal that putrescine is essential for parasite proliferation as putrescine-depleted $\Delta o d c$ mutants displayed an immediate cessation of proliferation, while putrescine-rich $\Delta s p d s y n$ mutants exhibited an intermediate proliferation phenotype compared to the $\Delta$ odc mutants (Fig. 3). As both cell lines contained similar, albeit low, amounts of spermidine (Fig. 4), the immediate cessation of proliferation in the $\Delta o d c$ mutants can be attributed to the lack of putrescine. In mammalian cells, polyamines have been linked to cellular proliferation (Bachrach et al. 2001; Igarashi and Kashiwagi 2000, 2010; Miller-Fleming et al. 2015; Minois et al. 2011; Pegg 2016); however, the contributions of putrescine, spermidine, or spermine are difficult to discern as mammalian cells have catabolic pathways that can convert spermine and spermidine to putrescine (Bachrach et al. 2001; Igarashi and Kashiwagi 2010; Seiler 2004; Seiler et al. 1981). Because Leishmania parasites lack a backconversion pathway to recover lost pools of putrescine, our results show for the first time an unambiguous function of putrescine for cellular proliferation.

As stated above, mutant parasites incubated in polyaminefree media contained spermidine (Fig. 4). Previous measurements of intracellular pools in $L$. donovani $\Delta o d c$ and $\Delta$ spdsyn knockout cell lines also showed that parasites maintained low but stable levels of spermidine over several days of starvation (Jiang et al. 1999; Roberts et al. 2001). Similarly, studies in L. major $\Delta$ arg mutants and arginine-depleted 
L. donovani wild-type parasites found a marked decrease of putrescine levels while spermidine pools diminished at a slower rate (Mandal et al. 2016; Reguera et al. 2009). Spermidine is an essential metabolite for the hypusination and activation of eIF5A (Chawla et al. 2010, 2012) and the formation of trypanothione (Colotti and Ilari 2011; Ilari et al. 2017). It is conceivable that less spermidine is being used for these downstream reactions to maintain spermidine pools. It is also possible that spermidine is formed from trypanothione as TRYS is a bifunctional enzyme catalyzing the biosynthesis and hydrolysis of trypanothione (Fyfe et al. 2008). Although, we have previously shown that trypanothione levels in starved $L$. donovani $\Delta o d c$ and $\Delta s p d s y n$ parasites indeed plummet over time (Jiang et al. 1999; Roberts et al. 2001), it is unknown whether this is due to a lack of trypanothione formation or conversion of trypanothione to spermidine. Regardless, the observation that polyaminestarved parasites maintain low but stable levels of spermidine underscore the importance of this polyamine.

Interestingly, a comparison of $\Delta o d c$ and $\Delta$ spdsyn parasites incubated in spermidine-supplemented media showed a profound difference in spermidine levels (Fig. 4), although both cell strains would have been expected to compensate for the loss of spermidine biosynthesis by increased uptake. While the $\Delta$ spdsyn mutants incubated in $100 \mu \mathrm{M}$ spermidine exhibited a twofold increase in spermidine levels compared to spermidine levels in wild-type cells, $\Delta o d c$ parasites incubated with $100 \mu \mathrm{M}, 500 \mu \mathrm{M}$, or $1000 \mu \mathrm{M}$ spermidine displayed spermidine levels that were lower than in wildtype parasites (Fig. 4). Thus, $\Delta s p d s y n$ parasites markedly increased spermidine uptake while $\Delta o d c$ parasites did not, which leads to intriguing speculations about the molecular mechanisms regulating spermidine transport. Perhaps, increased transport of spermidine in $\Delta s p d s y n$ parasites is regulated by loss of SPDSYN activity or metabolite flux rather than levels of metabolite pools. This conjecture is supported by a previous study on arginine transport in $L$. donovani $\Delta o d c$ and $\Delta s p d s y n$ parasites, which showed a significant reduction in arginine transport, even in the presence of putrescine or spermidine (Darlyuk et al. 2009). An alternative explanation for our observation that $\Delta o d c$ parasites exhibited only little increase in uptake of spermidine may be that the $\Delta o d c$ parasites, after 3 days of starvation, were already compromised in health and were thus not capable of robust uptake of spermidine.

Differences in morphology and motility between the two mutant strains were also substantial. While the starved $\Delta o d c$ parasites rounded up and clumped together within a few days, the starved $\Delta$ spdsyn mutants exhibited morphology similar to that of wild-type parasites and supplemented mutants throughout several days of starvation (Fig. 5). The observation that putrescine depletion caused this severe stress phenotype further supports our hypothesis that putrescine fulfills important roles in parasites.

Both $\Delta o d c$ and $\Delta s p d s y n$ mutants perished in media without the supplementation of putrescine or spermidine, respectively, although at different times. The $\Delta o d c$ parasites showed reduced viability within a few days (Fig. 6) and died after 2 weeks (Table 2). It is likely that the small amount of intracellular spermidine present after 3 days of starvation was depleted after 2 weeks, and that the lack of both polyamines caused cell death. In contrast, $\Delta s p d s y n$ parasites maintained a high level of viability (over 70\%), during 8 days of starvation (Fig. 6), and were able to survive for an average of 6 weeks before cell death occurred (Table 2). The presence of putrescine alone appeared to be sufficient to allow $\Delta s p d s y n$ parasites to enter a quiescentlike state. Surprisingly, the addition of $100 \mu \mathrm{M}$ spermidine to $\Delta o d c$ parasites enabled the mutants to survive for 14 weeks (Table 2). Thus, although the supplementation of $\Delta o d c$ parasites with spermidine had only a marginal effect on proliferation (Fig. 3) or initial viability (Fig. 6), it had a profound effect on parasite survival. Further experiments are necessary to quantitate differences in cell death in these cultures, and to determine what types of adaptations or mutations allowed persistence. Spermidine has been implied to have a role in longevity in mammalian cells, yeast, and nematodes (Madeo et al. 2010; Morselli et al. 2009; Petrovski and Das 2010), and polyamines may have a similar effect in Leishmania parasites. Thus, while both polyamines were essential for ultimate parasite survival, the presence of either putrescine or spermidine alone may allow parasites to survive for several weeks in a quiescent-like state. The intracellular mechanism and reprogramming associated with this phenomenon could be of clinical importance, as Leishmania parasites may also persist in infected patients (Bogdan 2008; Mandell and Beverley 2017). While some studies have investigated how the human immune system suppresses parasite numbers, very few investigations have explored how the parasite is able to persist.

It is of interest to note that the proliferation and survival discrepancies between the $\Delta o d c$ and $\Delta s p d s y n$ mutants mirrored in vivo infectivity phenotypes. While $\Delta o d c$ parasites exhibit profoundly diminished infectivity in mice compared to wild-type parasites (Boitz et al. 2009), the $\Delta$ spdsyn parasites show a less pronounced, although significant, reduction in intracellular survival (Gilroy et al. 2011). We previously postulated that putrescine salvage is severely limited in the phagolysosome (Boitz et al. 2016). Together, these observations suggest that putrescine is a key metabolite for both promastigotes and intracellular amastigotes, and validate the polyamine biosynthetic enzyme ODC as a promising therapeutic target. 


\section{Conclusions}

Collectively, our observations confirm a previous report that putrescine is not merely a precursor metabolite for spermidine formation and, furthermore, suggest that putrescine has specific functions for parasite proliferation and viability. While the polyamine biosynthetic pathway has already been endorsed as a potential therapeutic target, our studies highlight ODC inhibition and putrescine depletion as the most promising strategy. In addition, our results suggest that both polyamines are essential for parasite survival but that the presence of either putrescine or spermidine alone may have allowed parasites to survive in a quiescent-like state for several weeks. The $\Delta o d c$ and $\Delta s p d s y n$ mutants are valuable model systems to not only study the functions of putrescine but also to elucidate processes vital for proliferation and for intracellular reprogramming associated with survival versus cell death, which may ultimately lead to new therapeutic strategies.

Acknowledgements This work was supported in part by Grant AI041622 from the National Institute of Allergy and Infectious Diseases and Pacific University School of Pharmacy Research Incentive Grants. We thank Amber Buhler for microscopy and analysis training, Jon Taylor for technical support, and Amber Buhler and Jon Taylor for critically reading of the manuscript and providing feedback.

\section{Compliance with ethical standards}

Conflict of interest The authors declare that they have no conflict of interest.

Research involving human participants and/or animals This research did not involve human participants or animals.

Informed consent None.

Open Access This article is distributed under the terms of the Creative Commons Attribution 4.0 International License (http://creativeco mmons.org/licenses/by/4.0/), which permits unrestricted use, distribution, and reproduction in any medium, provided you give appropriate credit to the original author(s) and the source, provide a link to the Creative Commons license, and indicate if changes were made.

\section{References}

Alvar J et al (2012) Leishmaniasis worldwide and global estimates of its incidence. PLoS One 7:e35671. https://doi.org/10.1371/journ al.pone.0035671

Babokhov P, Sanyaolu AO, Oyibo WA, Fagbenro-Beyioku AF, Iriemenam NC (2013) A current analysis of chemotherapy strategies for the treatment of human African trypanosomiasis. Pathog Glob Health 107:242-252. https://doi.org/10.1179/2047773213 Y.0000000105

Bacchi CJ, McCann PP (1987) Parasitic protozoa and polyamines. In: McCann PP, Pegg AE, Sjoerdsma A (eds) Inhibition of polyamine metabolism: biological significance and basis for new therapies. Academic Press, Orlando, pp 317-344

Bachrach U, Wang YC, Tabib A (2001) Polyamines: new cues in cellular signal transduction. News Physiol Sci 16:106-109

Battaglia V, DeStefano Shields C, Murray-Stewart T, Casero RA Jr (2014) Polyamine catabolism in carcinogenesis: potential targets for chemotherapy and chemoprevention. Amino Acids 46:511-519. https://doi.org/10.1007/s00726-013-1529-6

Bern C, Maguire JH, Alvar J (2008) Complexities of assessing the disease burden attributable to leishmaniasis. PLoS Negl Trop Dis 2:e313. https://doi.org/10.1371/journal.pntd.0000313

Birkholtz LM, Williams M, Niemand J, Louw AI, Persson L, Heby $\mathrm{O}$ (2011) Polyamine homoeostasis as a drug target in pathogenic protozoa: peculiarities and possibilities. Biochem J 438:229 244. https://doi.org/10.1042/BJ20110362

Bisceglia JA, Mollo MC, Gruber N, Orelli LR (2018) Polyamines and related nitrogen compounds in the chemotherapy of neglected diseases caused by kinetoplastids. Curr Top Med Chem 18:321-368. https://doi.org/10.2174/156802661866618 0427151338

Bitonti AJ, McCann PP, Sjoerdsma A (1987) Plasmodium falciparum and Plasmodium berghei: effects of ornithine decarboxylase inhibitors on erythrocytic schizogony. Exp Parasitol 64:237-243

Bogdan C (2008) Mechanisms and consequences of persistence of intracellular pathogens: leishmaniasis as an example. Cell Microbiol 10:1221-1234. https://doi.org/10.1111/j.1462-5822.2008.01146.x

Boitz JM, Yates PA, Kline C, Gaur U, Wilson ME, Ullman B, Roberts SC (2009) Leishmania donovani ornithine decarboxylase is indispensable for parasite survival in the mammalian host. Infect Immun 77:756-763. https://doi.org/10.1128/IAI.01236-08

Boitz JM et al (2016) Arginase is essential for survival of Leishmania donovani promastigotes but not intracellular amastigotes. Infect Immun. https://doi.org/10.1128/iai.00554-16

Burri C, Brun R (2003) Eflornithine for the treatment of human African trypanosomiasis. Parasitol Res 90(Supp 1):S49-S52. https://doi. org/10.1007/s00436-002-0766-5

Casero RA Jr, Marton LJ (2007) Targeting polyamine metabolism and function in cancer and other hyperproliferative diseases. Nat Rev Drug Discov 6:373-390. https://doi.org/10.1038/nrd2243

Chawla B et al (2010) Identification and characterization of a novel deoxyhypusine synthase in Leishmania donovani. J Biol Chem 285:453-463. https://doi.org/10.1074/jbc.M109.048850

Chawla B, Kumar RR, Tyagi N, Subramanian G, Srinivasan N, Park MH, Madhubala R (2012) A unique modification of the eukaryotic initiation factor $5 \mathrm{~A}$ shows the presence of the complete hypusine pathway in Leishmania donovani. PLoS One 7:e33138. https:// doi.org/10.1371/journal.pone. 0033138

Colotti G, Ilari A (2011) Polyamine metabolism in Leishmania: from arginine to trypanothione. Amino Acids 40:269-285. https://doi. org/10.1007/s00726-010-0630-3

Croft SL, Sundar S, Fairlamb AH (2006) Drug resistance in leishmaniasis. Clin Microbiol Rev 19:111-126. https://doi.org/10.1128/ CMR.19.1.111-126.2006

da Silva MF, Floeter-Winter LM (2014) Arginase in Leishmania. Subcell Biochem 74:103-117. https://doi. org/10.1007/978-94-007-7305-9_4

da Silva MF, Zampieri RA, Muxel SM, Beverley SM, Floeter-Winter LM (2012) Leishmania amazonensis arginase compartmentalization in the glycosome is important for parasite infectivity. PLoS One 7:e34022. https://doi.org/10.1371/journal.pone.0034022

Darlyuk I, Goldman A, Roberts SC, Ullman B, Rentsch D, Zilberstein D (2009) Arginine homeostasis and transport in the human pathogen Leishmania donovani. J Biol Chem 284:19800-19807. https ://doi.org/10.1074/jbc.M901066200 
Docampo R, Moreno SN (2003) Current chemotherapy of human African trypanosomiasis. Parasitol Res 90(Supp 1):S10-S13. https:// doi.org/10.1007/s00436-002-0752-y

El-Sayed NM et al (2005) Comparative genomics of trypanosomatid parasitic protozoa. Science 309:404-409. https://doi.org/10.1126/ science. 1112181

Fairlamb AH (2003) Chemotherapy of human African trypanosomiasis: current and future prospects. Trends Parasitol 19:488-494

Fyfe PK, Oza SL, Fairlamb AH, Hunter WN (2008) Leishmania trypanothione synthetase-amidase structure reveals a basis for regulation of conflicting synthetic and hydrolytic activities. J Biol Chem 283:17672-17680. https://doi.org/10.1074/jbc.M801850200

Gaur U, Roberts SC, Dalvi RP, Corraliza I, Ullman B, Wilson ME (2007) An effect of parasite-encoded arginase on the outcome of murine cutaneous leishmaniasis. J Immunol 179:8446-8453

Gillin FD, Reiner DS, McCann PP (1984) Inhibition of growth of Giardia lamblia by difluoromethylornithine, a specific inhibitor of polyamine biosynthesis. J Protozool 31:161-163

Gilroy C, Olenyik T, Roberts SC, Ullman B (2011) Spermidine synthase is required for virulence of Leishmania donovani. Infect Immun 79:2764-2769. https://doi.org/10.1128/IAI.00073-11

Goyard S, Segawa H, Gordon J, Showalter M, Duncan R, Turco SJ, Beverley SM (2003) An in vitro system for developmental and genetic studies of Leishmania donovani phosphoglycans. Mol Biochem Parasitol 130:31-42

Gradoni L, Iorio MA, Gramiccia M, Orsini S (1989) In vivo effect of eflornithine (DFMO) and some related compounds on Leishmania infantum preliminary communication. Farmaco 44:1157-1166

Heby O, Roberts SC, Ullman B (2003) Polyamine biosynthetic enzymes as drug targets in parasitic protozoa. Biochem Soc Trans 31:415-419. https://doi.org/10.1042/bst0310415

Heby O, Persson L, Rentala M (2007) Targeting the polyamine biosynthetic enzymes: a promising approach to therapy of African sleeping sickness, Chagas' disease, and leishmaniasis. Amino Acids 33:359-366. https://doi.org/10.1007/s00726-007-0537-9

Igarashi K, Kashiwagi K (2000) Polyamines: mysterious modulators of cellular functions. Biochem Biophys Res Commun 271:559-564. https://doi.org/10.1006/bbrc.2000.2601

Igarashi K, Kashiwagi K (2010) Modulation of cellular function by polyamines. Int J Biochem Cell Biol 42:39-51. https://doi. org/10.1016/j.biocel.2009.07.009

Ilari A, Fiorillo A, Baiocco P, Poser E, Angiulli G, Colotti G (2015) Targeting polyamine metabolism for finding new drugs against leishmaniasis: a review. Mini Rev Med Chem 15:243-252

Ilari A, Fiorillo A, Genovese I, Colotti G (2017) Polyamine-trypanothione pathway: an update. Future Med Chem 9:61-77. https:// doi.org/10.4155/fmc-2016-0180

Iovannisci DM, Ullman B (1983) High efficiency plating method for Leishmania promastigotes in semidefined or completely-defined medium. J Parasitol 69:633-636

Jiang Y et al (1999) Ornithine decarboxylase gene deletion mutants of Leishmania donovani. J Biol Chem 274:3781-3788

Kahana C (2018) The antizyme family for regulating polyamines. J Biol Chem 293:18730-18735. https://doi.org/10.1074/jbc.TM118 .003339

Kaur K, Emmett K, McCann PP, Sjoerdsma A, Ullman B (1986) Effects of DL-alpha-difluoromethylornithine on Leishmania donovani promastigotes. J Protozool 33:518-521

Kaye P, Scott P (2011) Leishmaniasis: complexity at the host-pathogen interface. Nat Rev Microbiol 9:604-615. https://doi.org/10.1038/ nrmicro2608

Kedzierski L (2011) Leishmaniasis. Hum Vaccines 7:1204-1214. https ://doi.org/10.4161/hv.7.11.17752

Kedzierski L, Sakthianandeswaren A, Curtis JM, Andrews PC, Junk PC, Kedzierska K (2009) Leishmaniasis: current treatment and prospects for new drugs and vaccines. Curr Med Chem 16:599-614

Krauth-Siegel RL, Comini MA (2008) Redox control in trypanosomatids, parasitic protozoa with trypanothione-based thiol metabolism. Biochimica et Biophysica Acta 1780:1236-1248. https:// doi.org/10.1016/j.bbagen.2008.03.006

Krauth-Siegel RL, Inhoff O (2003) Parasite-specific trypanothione reductase as a drug target molecule. Parasitol Res 90(Suppl 2):S77-S85. https://doi.org/10.1007/s00436-002-0771-8

Krauth-Siegel RL, Meiering SK, Schmidt H (2003) The parasite-specific trypanothione metabolism of trypanosoma and leishmania. Biol Chem 384:539-549. https://doi.org/10.1515/BC.2003.062

Krauth-Siegel LR, Comini MA, Schlecker T (2007) The trypanothione system. Subcell Biochem 44:231-251

Lenis YY, Elmetwally MA, Maldonado-Estrada JG, Bazer FW (2017) Physiological importance of polyamines. Zygote 25:244-255. https://doi.org/10.1017/S0967199417000120

Madeo F, Eisenberg T, Buttner S, Ruckenstuhl C, Kroemer G (2010) Spermidine: a novel autophagy inducer and longevity elixir. Autophagy 6:160-162

Mandal A et al (2016) Deprivation of L-arginine induces oxidative stress mediated apoptosis in Leishmania donovani promastigotes: contribution of the polyamine pathway. PLoS Negl Trop Dis 10:e0004373. https://doi.org/10.1371/journal.pntd.0004373

Mandell MA, Beverley SM (2017) Continual renewal and replication of persistent Leishmania major parasites in concomitantly immune hosts. Proc Natl Acad Sci USA 114:E801-E810. https ://doi.org/10.1073/pnas.1619265114

Manta B, Comini M, Medeiros A, Hugo M, Trujillo M, Radi R (2013) Trypanothione: a unique bis-glutathionyl derivative in trypanosomatids. Biochim Biophys Acta 1830:3199-3216. https ://doi.org/10.1016/j.bbagen.2013.01.013

Manta B, Bonilla M, Fiestas L, Sturlese M, Salinas G, Bellanda M, Comini MA (2018) Polyamine-based thiols in trypanosomatids: evolution, protein structural adaptations, and biological functions. Antioxid Redox Signal 28:463-486. https://doi. org/10.1089/ars.2017.7133

Miller-Fleming L, Olin-Sandoval V, Campbell K, Ralser M (2015) Remaining mysteries of molecular biology: the role of polyamines in the cell. J Mol Biol 427:3389-3406. https://doi. org/10.1016/j.jmb.2015.06.020

Minois N, Carmona-Gutierrez D, Madeo F (2011) Polyamines in aging and disease. Aging (Albany NY) 3:716-732. https://doi. org/10.18632/aging.100361

Mishra J, Saxena A, Singh S (2007) Chemotherapy of leishmaniasis: past, present and future. Curr Med Chem 14:1153-1169

Morselli E et al (2009) Autophagy mediates pharmacological lifespan extension by spermidine and resveratrol. Aging (Albany NY) 1:961-970. https://doi.org/10.18632/aging.100110

Mukhopadhyay R, Madhubala R (1993) Effect of a bis(benzyl) polyamine analogue, and DL-alpha-difluoromethylornithine on parasite suppression and cellular polyamine levels in golden hamster during Leishmania donovani infection. Pharmacol Res 28:359-365. https://doi.org/10.1006/phrs.1993.1138

Muleme HM et al (2009) Infection with arginase-deficient Leishmania major reveals a parasite number-dependent and cytokineindependent regulation of host cellular arginase activity and disease pathogenesis. J Immunol 183:8068-8076. https://doi. org/10.4049/jimmunol.0803979

Muller R (2007) Advances in parasitology, vol 65. Academic Press, San Diego

Murray-Stewart TR, Woster PM, Casero RA Jr (2016) Targeting polyamine metabolism for cancer therapy and prevention. Biochem J 473:2937-2953. https://doi.org/10.1042/BCJ20160383

Olenyik T, Gilroy C, Ullman B (2011) Oral putrescine restores virulence of ornithine decarboxylase-deficient Leishmania 
donovani in mice. Mol Biochem Parasitol 176:109-111. https ://doi.org/10.1016/j.molbiopara.2010.12.004

Park MH, Wolff EC (2018) Hypusine, a polyamine-derived amino acid critical for eukaryotic translation. J Biol Chem 293:18710 18718. https://doi.org/10.1074/jbc.tm118.003341

Pegg AE (2016) Functions of polyamines in mammals. J Biol Chem 291:14904-14912. https://doi.org/10.1074/jbc.R116.731661

Petrovski G, Das DK (2010) Does autophagy take a front seat in lifespan extension? J Cell Mol Med 14:2543-2551. https://doi.org/10 $.1111 / j .1582-4934.2010 .01196 . x$

Phillips MA (2018) Polyamines in protozoan pathogens. J Biol Chem 293:18746-18756. https://doi.org/10.1074/jbc.TM118.003342

Ponte-Sucre A et al (2017) Drug resistance and treatment failure in leishmaniasis: a 21st century challenge. PLoS Negl Trop Dis 11:e0006052. https://doi.org/10.1371/journal.pntd.0006052

Reguera RM, Balana-Fouce R, Showalter M, Hickerson S, Beverley SM (2009) Leishmania major lacking arginase (ARG) are auxotrophic for polyamines but retain infectivity to susceptible BALB/c mice. Mol Biochem Parasitol 165:48-56. https://doi. org/10.1016/j.molbiopara.2009.01.001

Roberts SC (2013) Genetic manipulation of Leishmania parasites facilitates the exploration of the polyamine biosynthetic pathway as a potential therapeutic target. In: Urbano KV (ed) Advances in genetics research, vol 10. Nova Science Publishers, Hauppauge, NY, pp 29-54

Roberts S, Ullman B (2017) Parasite polyamines as pharmaceutical targets. Curr Pharm Des 23:3325-3341. https://doi. org/10.2174/1381612823666170601101644

Roberts SC, Jiang Y, Jardim A, Carter NS, Heby O, Ullman B (2001) Genetic analysis of spermidine synthase from Leishmania donovani. Mol Biochem Parasitol 115:217-226

Roberts SC et al (2002) S-Adenosylmethionine decarboxylase from Leishmania donovani. Molecular, genetic, and biochemical characterization of null mutants and overproducers. J Biol Chem 277:5902-5909. https://doi.org/10.1074/jbc.M110118200
Roberts SC, Tancer MJ, Polinsky MR, Gibson KM, Heby O, Ullman B (2004) Arginase plays a pivotal role in polyamine precursor metabolism in Leishmania. Characterization of gene deletion mutants. J Biol Chem 279:23668-23678. https://doi.org/10.1074/ jbc.M402042200

Seiler N (2004) Catabolism of polyamines. Amino Acids 26:217-233. https://doi.org/10.1007/s00726-004-0070-z

Seiler N, Bolkenius FN, Rennert OM (1981) Interconversion, catabolism and elimination of the polyamines. Med Biol 59:334-346

Shim H, Fairlamb AH (1988) Levels of polyamines, glutathione and glutathione-spermidine conjugates during growth of the insect trypanosomatid Crithidia fasciculata. J Gen Microbiol 134:807817. https://doi.org/10.1099/00221287-134-3-807

Singh N, Kumar M, Singh RK (2012) Leishmaniasis: current status of available drugs and new potential drug targets. Asian Pac J Trop Med 5:485-497. https://doi.org/10.1016/s1995-7645(12)60084-4

Tavladoraki P et al (2012) Polyamine catabolism: target for antiproliferative therapies in animals and stress tolerance strategies in plants. Amino Acids 42:411-426. https://doi.org/10.1007/s0072 6-011-1012-1

Wang Y, Casero RA Jr (2006) Mammalian polyamine catabolism: a therapeutic target, a pathological problem, or both? J Biochem 139:17-25. https://doi.org/10.1093/jb/mvj021

Xiao Y, McCloskey DE, Phillips MA (2009) RNA interference-mediated silencing of ornithine decarboxylase and spermidine synthase genes in Trypanosoma brucei provides insight into regulation of polyamine biosynthesis. Eukaryot Cell 8:747-755. https://doi. org/10.1128/EC.00047-09

Publisher's Note Springer Nature remains neutral with regard to jurisdictional claims in published maps and institutional affiliations. 